\title{
Genetic pathway to recurrent chromosome translocations in murine lymphoma involves $\mathrm{V}(\mathrm{D}) \mathrm{J}$ recombinase
}

\author{
Gary J. Vanasse, ${ }^{1,4}$ James Halbrook, ${ }^{2}$ Sushma Thomas, ${ }^{3}$ Abigail Burgess, ${ }^{4}$ \\ Merl F. Hoekstra, ${ }^{2}$ Christine M. Disteche, ${ }^{3}$ and Dennis M. Willerford ${ }^{1,4}$ \\ ${ }^{1}$ Department of Medicine, University of Washington, Seattle, Washington 98195, USA
2ICOS Corporation, Bothell, Washington 98021, USA
${ }^{3}$ Department of Pathology, University of Washington, Seattle, Washington 98195, USA
${ }^{4}$ Puget Sound Blood Center, Seattle, Washington 98104, USA \\ Address correspondence to: Dennis M. Willerford, Puget Sound Blood Center, 921 Terry Avenue, Seattle, Washington 98104, USA. \\ Phone: (206) 292-7077; Fax: (206) 343-1776; E-mail: dwiller@u.washington.edu.
}

Received for publication February 26, 1999, and accepted in revised form May 10, 1999.

\begin{abstract}
Chromosome translocations involving antigen receptor loci are a genetic hallmark of non-Hodgkin's lymphomas in humans. Most commonly, these translocations result in juxtaposition of the immunoglobulin heavy-chain ( $\mathrm{IgH})$ locus with one of several cellular proto-oncogenes, leading to deregulated oncogene expression. The $\mathrm{V}(\mathrm{D}) \mathrm{J}$ recombinase, which mediates physiologic rearrangements of antigen receptor genes, may play a mechanistic role in some lymphoma translocations, although evidence is indirect. A high incidence of B-lineage lymphomas has been observed in mice with severe combined immunodeficiency (SCID) and p53-null mutations. We show that these tumors are characteristic of the pro-B-cell stage of development and that they harbor recurrent translocations involving chromosomes 12 and 15. Fluorescence in situ hybridization (FISH) shows retention of IgH sequences on the derivative chromosome 12, implying that breakpoints involve the IgH locus. Pro-B-cell lymphomas were suppressed in SCID $p 53^{-/-}$mice by a Rag-2-null mutation, demonstrating that DNA breaks generated during $\mathrm{V}(\mathrm{D}) \mathrm{J}$ recombination are required for oncogenic transformation, and suggesting that $\mathrm{t}(12 ; 15)$ arise during attempted IgH rearrangement in pro-B cells. These studies indicate that the oncogenic potential inherent in antigen receptor diversification is controlled in vivo by efficient rejoining of DNA ends generated during $\mathrm{V}(\mathrm{D}) \mathrm{J}$ recombination and an intact cellular response to DNA damage.
\end{abstract}

J. Clin. Invest. 103:1669-1675 (1999).

\section{Introduction}

Chromosome translocations involving antigen receptor genes represent a central genetic abnormality in the pathogenesis of lymphoid malignancies in humans, particularly the non-Hodgkin's lymphomas (1-4). Transposition of a cellular proto-oncogene in proximity to the regulatory elements of antigen receptor loci leads to deregulation of oncogene expression, with subsequent effects on cell growth, differentiation, or apoptosis. The involvement of antigen receptor genes in lymphoma translocations parallels physiologic mechanisms of genome rearrangement in lymphocytes. $\mathrm{V}(\mathrm{D}) \mathrm{J}$ recombination joins immunoglobulin and T-cell receptor (TCR) variable-region gene segments to generate the vast diversity of antigen specificities characteristic of vertebrate immunity (reviewed in refs. 5, 6). Peripheral B cells may also undergo isotype switch recombination within the IgH locus, substituting production of $\operatorname{IgG}$, IgE, or IgA for IgM and IgD (7). Circumstantial evidence suggests that in some cases translocations may be catalyzed by the $V(D) J$ or switch recombinases, based on similarities between breakpoint sequences and physiologic $\mathrm{V}(\mathrm{D}) \mathrm{J}$ or switch joints (8-13). However, the structure of many breakpoints is not characteristic of a particular rearrangement process, and a thorough understanding of the cause of lymphoma translocations is lacking.
Much has been learned in the past few years regarding the mechanism of $\mathrm{V}(\mathrm{D}) \mathrm{J}$ recombination. DNA scission is catalyzed by the Rag- 1 and Rag- 2 proteins at recognition signals (RSS) bordering antigen receptor variable-region gene segments, creating blunt signal ends and sealed hairpins at coding ends (14-17). Mice and humans with induced null mutations of Rag-1 or Rag- 2 are unable to initiate rearrangement of endogenous Ig or TCR loci and consequently lack mature B or T cells (18-20). Rejoining of coding ends requires the DNA-dependent protein kinase (DNA-PK) complex, including the catalytic subunit (DNA-PKcs), Ku70 and Ku86, whereas signal ends are resolved by a distinct pathway, also involving the $\mathrm{Ku}$ proteins (reviewed in refs. 6, 21). Severe combined immunodeficiency (SCID) mice, which have a mutation affecting the DNA-PKcs, are capable of initiating Ragmediated DNA cleavage but are severely limited in their ability to efficiently rejoin coding ends. As a result, there is a near-complete arrest of T- and B-cell development at an early progenitor stage, with accumulation of unresolved DNA breaks at coding ends (22-26).

The p53 tumor suppressor gene is mutated in a wide variety of human cancers and plays an important role in the cellular response to DNA damage. DNA breaks lead to rapid upregulation of $\mathrm{p} 53$ expression, which leads to 2 major cellular effects: arrest of the cell cycle at the G1 
phase and induction of apoptosis (27-29). Mice lacking p53 are susceptible to several types of malignancies; of these, up to two thirds are lymphomas, which are predominantly localized thymic tumors $(30,31)$. SCID mice are also susceptible to thymic lymphomas, which may occur in up to $15 \%$ of animals (23). Mice with combined SCID and p53-null mutations develop disseminated Bcell lymphomas with an incidence approaching $100 \%$; these lymphomas occur at a younger age than do lymphoid tumors in either parental strain (31-33). In this report we show that pro-B-cell lymphomas in SCID $p 53^{-1-}$ mice harbor recurrent translocations involving the telomere of chromosome 12, at or near the IgH locus, and that tumor development requires initiation of $\mathrm{V}(\mathrm{D}) \mathrm{J}$ recombination. This genetic pathway to recurrent lymphoma translocations indicates that mitigation of the oncogenic potential inherent in antigen receptor gene rearrangement requires efficient rejoining of cleaved DNA and an intact cellular response to DNA damage.

\section{Methods}

Mice. SCID $p 53^{+/-}$mice (ICR and C57Bl/ 6 backgrounds; Taconic Farms, Germantown, New York, USA) were intercrossed to generate SCID $p 53^{-/-}$mice. Rag-2-/- mice (C57Bl/ 6 and 129 backgrounds; obtained from Frederick Alt, Children's Hospital,

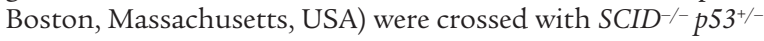
mice, and offspring were bred to generate the following 3 experimental cohorts: $p 53^{-/-}\left(S C I D^{w t / w t}, \operatorname{Rag}_{-2^{+/-}}\right.$, or $\left.\mathrm{Rag}_{-2^{+/+}}\right)$; SCID

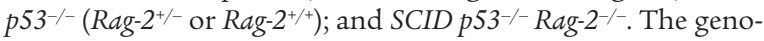
type of all mice was confirmed by PCR for each allele. Mice were housed under specific pathogen-free conditions and sacrificed upon recognition of tumor masses or ill health, which marked the endpoint for calculating tumor incidence. Kaplan-Meier analysis was performed using the SPSS software package, and separate analyses were performed for mortality due to disseminated pro-B-cell lymphomas and for all tumors. Statistical analysis of survival differences was performed using the Wilcoxon log rank test.

Analysis of tumor phenotype. Tumor tissue was fixed in formalin for standard histology. For flow cytometry, cell suspensions were stained using FITC-, phycoerythrin-, biotin-, or allophycocyanin-conjugated antibodies to the indicated markers, with secondary staining by streptavidin-CyChrome (PharMingen, San Diego, California, USA). Analysis was performed using a dual-laser FACScalibur (Becton Dickinson Immunocytometry Systems, San Jose, California, USA). Cytospin preparations from primary tumors were fixed in ice-cold acetone and stained using horseradish peroxidase-conjugated goat anti-mouse $\operatorname{IgM}, \mu$-chain specific, followed by colorimetric detection using 3, 3'-diaminobenzidine (Sigma Chemical Co., St. Louis, Missouri, USA) according to the manufacturer's protocol. Controls
Table 1

Southern blot analysis of $\mathrm{IgH}$ rearrangements in SCID $p 53^{-/-}$and $p 53^{-/-}$ lymphomas

\begin{tabular}{|c|c|c|c|c|c|c|}
\hline Number & Genotype & $\mathrm{JH}$ & $\mathrm{JH}$ & $\mathrm{C} \mu$ & $\mathrm{C} \mu$ & $\mathrm{C} \delta$ \\
\hline Sp02 & SCID $p 53^{-/-}$ & $\mathrm{R}$ & G & G & * & \\
\hline $\mathrm{Sp} 03$ & SCID $p 53^{-/-}$ & $\mathrm{R}$ & Del & G & $\mathrm{R}$ & G \\
\hline Sp04 & SCID $p 53^{-/-}$ & $\mathrm{R}$ & Del & G & $\mathrm{R}$ & \\
\hline Sp05 & SCID $p 53^{-/-}$ & G & * & G & * & \\
\hline Sp06 & SCID p $53^{-/-}$ & Del & Del & G & $\mathrm{R}$ & G \\
\hline Sp07 & SCID $p 53^{-/-}$ & G & Del & G & $\mathrm{R}$ & G \\
\hline Sp08 & SCID $p 53^{-/-}$ & $\mathrm{R}$ & Del & G & Del & G \\
\hline P01 & $P 53^{-/-}$ & $\mathrm{R}$ & G & G & * & \\
\hline P02 & P53--- & $\mathrm{R}$ & G & G & * & \\
\hline
\end{tabular}

Genomic digests using EcoRI, BamHI, and Bg/ll were analyzed using the indicated probes, except for $C \delta$, in which $E c o R I$ and $B a m H I$ digests were analyzed. For each probe, the status of both alleles is indicated. G, germ line; R, rearranged; Del, deleted. For germ-line bands in which the status of the second allele could not be determined (i.e., either germ line or deleted), the second allele is denoted by an asterisk.

included normal mouse spleen cells, murine cell lines p5424 (pro-T cell), and WEHI 231.1 (immature B cell).

Southern blot analysis. Genomic DNA was digested with EcoRI, BamHI, or BglII and resolved by agarose gel electrophoresis prior to transfer to nylon membranes. Probes were used to detect rearrangements involving JH (34), $\mathrm{C} \mu$ (600-bp cDNA fragment starting near the $5^{\prime}$ end of the constant region), and C $\delta$ (500-bp genomic DNA fragment encompassing the first exon). A genomic library was made from the SP04 tumor by ligation of partial Sau3A-digested genomic DNA into the sCOS1 vector, followed by packaging with lambda extracts, according to the manufacturer's instructions (Stratagene, La Jolla, California, USA). The library was grown on replica filters and screened with the $\mathrm{JH}$ probe using standard techniques.

Fluorescence in situ bybridization analysis. Metaphase spreads were prepared from primary lymphoma cell suspensions incubated for 2-6 hours with colchicine, and G-band analysis was performed using standard techniques. Chromosome 12 and 15 DNA paints (Oncor Inc., Gaithersburg, Maryland, USA) were labeled with digoxigenin and biotin, respectively, and were hybridized using the manufacturer's protocol. Fluorescence in situ hybridization (FISH) probes included a BAC clone covering the constant regions of the IgH locus (generous gift of Roy Riblet, Medical Biology Institute, La Jolla, California, USA), a c-myc cosmid probe generated in our laboratory, and a P1 clone identifying the centromeric region of mouse chromosome 12 (35). FISH was performed as previously described (36), after labeling probes with biotin or digoxigenin by nick translation (GIBCO BRL, Rockville, Maryland, USA). Detection used fluorescein- or Texas red-conjugated avidin and fluorescein- or rhodamine-conjugated anti-digoxigenin (Vector Laboratories, Burlingame, California, USA). Chromosomes were identified with 4',6'-diamidino-2-phenylindole (DAPI) staining.

\section{Figure 1}

Flow cytometric analysis of primary tumor cells from lymphomas arising in $p 53^{-/-}$and SCID $p 53^{-/-}$mice. The majority of $p 53^{-/-}$tumors were thymic lymphomas with T-cell markers (top row, representative of 5 tumors), whereas 1 peripheral B-cell lymphoma expressing IgM was observed (mid-

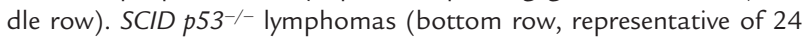
tumors) expressed B-lineage markers but lacked IgM expression. Singlecell suspensions from primary tumors were stained with antibodies to the markers indicated. Fluorescence intensity is expressed on a logarithmic scale. Control (unstained) plots are shown in broken lines.

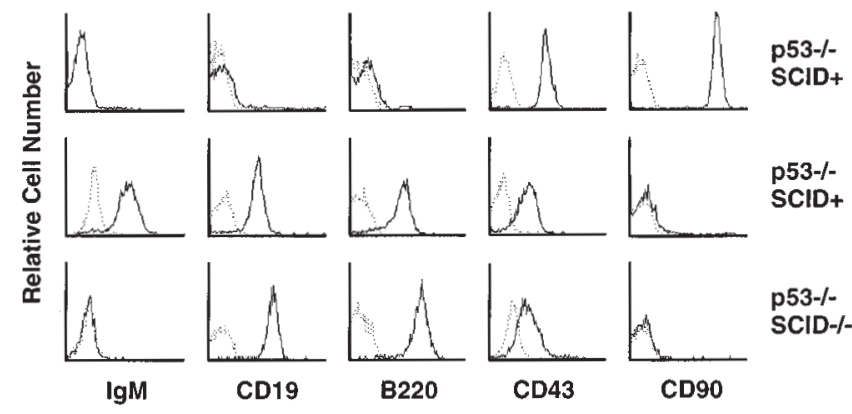




\section{Figure 2}

G-band karyotype showing chromosome 12 from a representative SCID $p 53^{-/-}$lymphoma. The normal chromosome is on the left. Arrow indicates a translocation.

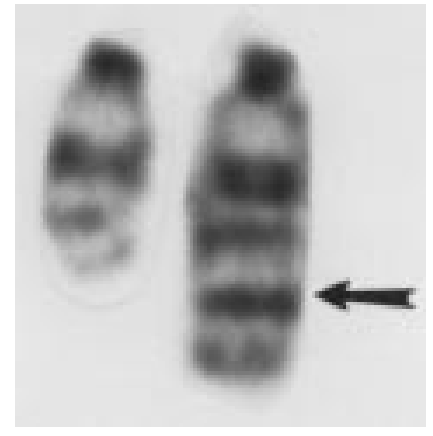

\section{Results}

SCID $p 53^{-1-}$ lymphomas have a pro-B-cell phenotype. In agreement with prior reports in the literature (31-33), we found that 24 of 24 SCID $p 53^{-/-}$mice developed lymphomas at 6-13 weeks of age, with lymphomas observed in 6 of $15 p 53^{-/-}$animals at later times. SCID mice had a significantly lower tumor incidence. Lymphomas in $p 53^{-1-}$ mice included 5 thymic lymphomas and 1 localized neck mass. In contrast, SCID $p 53^{-/-}$tumors were disseminated throughout lymphoid tissue and other organs, including kidney and liver. Peripheral blood films demonstrated that lymphomas in 6 of 6 SCID $p 53^{-/-}$mice were associated with frank leukemia, a finding that was absent in 2 of $2 p 53^{-/-}$lymphomas studied. Histologic examination of SCID $p 53^{-/-}$tumor tissue revealed near-total replacement of normal lymphoid architecture with high-grade lymphoma.

Flow cytometric analysis of 24 SCID $p 53^{-/-}$lymphomas revealed expression of B-cell markers B220 and CD19, but not T-cell markers CD4, CD8, or CD90 (Figure 1). These tumors were negative for surface IgM and showed low to moderate expression of CD 43. Six $p 53^{-/-}$tumors were similarly characterized and included 5 thymic tumors expressing T-cell markers CD4, CD8, and CD90, which had either negative (1 tumor), low ( 3 tumors), or high (1 tumor) expression of TCR- $\alpha \beta$. One cervical lymphoma in a $p 53^{-1-}$ mouse was positive for B220, CD19, and surface IgM, indicating a mature stage of B-cell development (Figure 1). Six SCID $p 53^{-/-}$tumors were also characterized for expression of cytoplasmic $\mu \mathrm{H}$ chain by immunohistochemistry on cytospin preparations, which was negative in all cases. Thus, $S C I D p 53^{-/-}$lymphomas most resemble the pro-B-cell developmental stage.

Bone marrow cells in the pro-B-cell compartment undergo sequential $\mathrm{D}$ to J rearrangements, followed by V-to-DJ rearrangements. If the latter are in frame, production of $\mu \mathrm{H}$ chain drives developmental progression to the pre-B-cell stage (37). To better characterize the stage of B-lineage development represented by SCID $p 53^{-/-}$lymphomas, rearrangements of the IgH locus were analyzed by genomic Southern blots of primary tumor DNA using a JH-region probe (Table 1). In addition, because rearrangements in SCID mice may exhibit large deletions (38), rearrangements within $\mathrm{C} \mu$ and $\mathrm{C} \delta$ were analyzed. Overall, $\mathrm{IgH}$ locus rearrangements were found in 6 of 7 SCID $p 53^{-/-}$tumors. In 4 of these, at least $1 \mathrm{JH}$ allele was rearranged. Tumor SP04 demon-

Table 2 strated rearrangement of $1 \mathrm{JH}$ allele and deletion of $\mathrm{JH}$ on the second allele, with rearrangement of $\mathrm{C} \mu$. Using a cosmid genomic library, the $\mathrm{JH}$ rearrangement from SP04 was cloned and analyzed by DNA sequencing, revealing a nonproductive rearrangement involving a $\mathrm{DH}$ pseudogene joined to JH4. Rearrangement of the JH probe was not detected with 2 different digests in 3 of 7 SCID $p 53^{-/-}$tumors. Two of these tumors exhibited rearrangement of $\mathrm{C} \mu$, indicating a large deletion involving JH on the second allele. Thus, in 4 of 4 SCID $p 53^{-/-}$ tumors in which rearrangements could be definitively characterized, we were able to exclude productive $V(D) J$ rearrangements. In $p 53^{-/-}$tumors we found JH rearrangements in 1 thymic lymphoma, likely representing DJ rearrangement, which may occur at some frequency in $\mathrm{T}$ cells, and in 1 IgM-expressing B-cell lymphoma, which reflects a productive $V(D) J$ rearrangement. These data indicate that the majority of lymphomas arising in SCID $p 53^{-/-}$mice are of a relatively uniform type of high-grade lymphoma representing the pro-B-cell stage of development, and they differ with respect to lineage or developmental stage from the thymic or mature B-cell lymphomas observed in our study or reported for the parental $p 53^{-/-}$strain (39).

SCID $p 5^{-1-}$ lymphomas carry recurrent chromosome 12 translocations. In humans, clinical and histologic subtypes of nonHodgkin's lymphoma correlate with specific chromosome translocations (40). To determine whether SCID $p 53^{-/-}$ pro-B-cell lymphomas were associated with recurrent cytogenetic abnormalities, we performed G-band karyotype studies on primary tumors from 7 SCID $p 53^{-/-}$mice and 3 $p 53^{-/-}$mice. In 6 of 7 SCID $p 53^{-/-}$lymphomas, additional genetic material was observed at the telomere of chromosome 12 , indicating a translocation (Figure 2 and Table 2). The band pattern suggested that this material was derived from chromosome 15 . To identify the translocation partner, dual-color FISH was used with DNA paints specific for chromosomes 12 and 15 (Figure $3 a$ and Table 2). In 5 of 6 tumors containing a chromosome 12 translocation, the partner was identified as chromosome 15 , whereas in the sixth tumor the chromosome 12 translocation involved a different partner. In contrast to these results, 3 lymphomas from $p 53^{-/-}$mice exhibited a hyperdiploid karyotype and

Cytogenetic analysis of SCID $p 53^{-/-}$and $p 53^{-/-}$lymphomas

$\begin{array}{lccccc}\text { Number } & \text { Genotype } & \text { Phenotype } & \text { Translocation } & \text { der12 breakpoint } & \text { Other abnormalities } \\ \text { Sp02 } & S C I D p 53^{-/-} & \text {Pro-B } & \text { ND } & \text { NA } & \operatorname{del}(2),+5 \\ \text { Sp03 } & S C I D p 53^{-/-} & \text {Pro-B } & \mathrm{t}(12 ; 15) & \text { IgH locus } & \text { add }(15) \\ \text { Sp04 } & S C I D p 53^{-/-} & \text {Pro-B } & \mathrm{t}(12 ; 15) & \text { Undetermined } & -11 \text {, add }(15),-16 \\ \text { Sp05 } & S C I D p 53^{-/-} & \text {Pro-B } & \mathrm{t}(12 ; 15) & \text { Undetermined } & +5 \text {, add }(15) \\ \text { Sp06 } & S C I D p 53^{-/-} & \text {Pro-B } & \mathrm{t}(12 ; 15) & \text { IgH locus } & +11 \text {, add }(15),-16 \\ \text { Sp07 } & S C I D p 53^{-/-} & \text {Pro-B } & \mathrm{t}(12 ; 15) & \text { IgH locus } & \text { add }(15),-19 \\ \text { Sp08 } & S C I D p 53^{-/-} & \text {Pro-B } & \mathrm{t}(12 ; ?) & \text { IgH locus } & +3,-8, \text { add }(18) \\ \text { P01 } & P 53^{-/-} & \text {Pre-T } & \text { ND } & \text { NA } & \text { Hyperdiploid } \\ \text { P02 } & P 53^{-/-} & \text {Mature B } & \text { ND } & \text { NA } & \text { Hyperdiploid } \\ \text { P03 } & P 53^{-/-} & \text {Pre-T } & \text { ND } & \text { NA } & \text { Hyperdiploid }\end{array}$

Tumor phenotype was assigned as described in the text. Translocations were identified using G band karyotype and dual-color FISH, with chromosome 12 and 15 paints as in Figure 3. The der12 was localized using an IgH BAC, where retention of this probe was interpreted as indicating an IgH locus breakpoint. G-band analysis on PO1, PO2, and PO3 tumors was incomplete because of suboptimal chromosome morphology; however, chromosome 12 translocations were not detected by visual inspection or by dual-color FISH analysis with paints specific for chromosomes 12 and 15 . ND, none detected; NA, not applicable. 

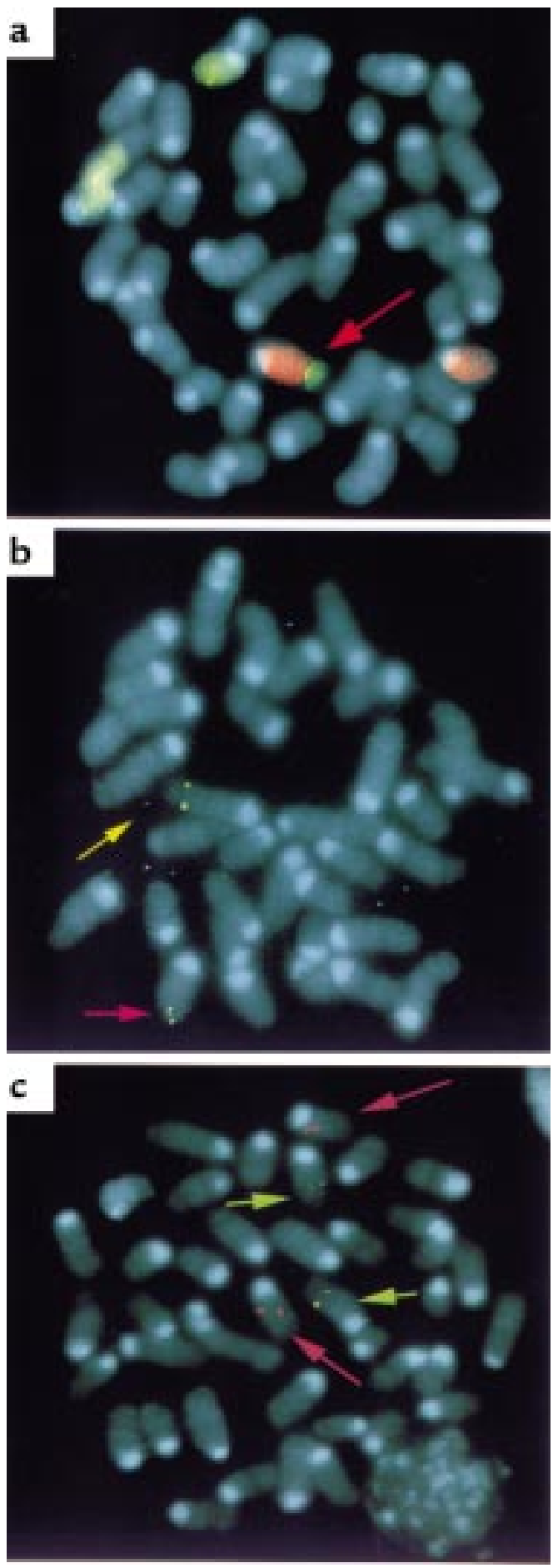

Figure 3

FISH analysis of representative SCID $p 53^{-/-}$primary tumor metaphase spreads. (a) Whole-chromosome paints specific for chromosomes 12 (red) and 15 (green). Red arrow shows t $12 ; 15)$, which was present in the majority of metaphases. Several tumors, including this one, demonstrated a large chromosome 15, possibly representing a duplication or amplification event. (b) IgH BAC probe. Hybridization is seen at the telomere on the normal chromosome 12 (red arrow) and at the breakpoint region on der12 (yellow arrow). Retention of the BAC signal on der12 indicates that the breakpoint lies within or telomeric to the region spanned by the probe. (c) Dual-color FISH analysis using chromosome 12 centromeric-region probe (red arrow) and c-myc probe (green arrow), demonstrating absence of c-myc on the der 12 chromosome. lacked $\mathrm{t}(12 ; 15)$, as determined by dual-color FISH analysis. The IgH locus extends to the telomere on human chromosome 14 (41) and is similarly positioned on mouse chromosome 12 , although continuity with the telomere has not yet been shown. By G-band analysis, the chromosome 12 breakpoint is consistent with the murine $\mathrm{IgH}$ complex. To further localize the breakpoint region, primary tumor metaphase spreads were hybridized with a BAC probe containing the $3^{\prime}$ part of the IgH locus, extending from the proximal $\mathrm{VH}$ segments to include $\mathrm{C} \alpha$. In 4 of 6 tumors analyzed, the IgH BAC signal was retained on the derivative chromosome 12 (der12; Figure $3 \mathrm{~b}$ and Table 2 ), implying that the breakpoint was within the IgH locus. Lack of an IgH signal in $2 \mathrm{t}(12 ; 15)$ tumors could indicate that the breakpoint is located toward the $3^{\prime}$ end of the IgH locus (where the BAC probe may have diminished sensitivity) or centromeric to the probe. We did not detect a reciprocal $t(15 ; 12)$, either with chromosome paints or with the IgH BAC probe. Taken together, these data show that SCID and p53-null mutations define a genetic pathway to pro-B-cell lymphomas that carry recurrent translocations involving chromosome 12 , probably within the IgH locus.

Review of the genetic map of chromosome 15 suggested c-myc as a candidate oncogene for involvement in $\mathrm{t}(12 ; 15)$ translocation. The position of $\mathrm{c}-\mathrm{myc}$ in $\mathrm{t}(12 ; 15)$ pro-B-cell lymphomas was analyzed using dual-color FISH with probes for c-myc and the centromeric region of chromosome 12 (Figure 3c). Whereas c-myc signals were readily recognized on chromosome 15 , they were absent from the der 12 chromosome. Similar results were obtained in 4 additional $\mathrm{t}(12 ; 15)$ tumors, indicating that $\mathrm{c}-m y c$ is not deregulated directly by the $\mathrm{t}(12 ; 15)$ translocations, and suggesting the involvement of another oncogene on chromosome 15.

Initiation of $V(D) J$ recombination is required for $t(12 ; 15)$ pro-B-cell lymphomas in SCID $p 53^{-1-}$ mice. Predisposition to $\mathrm{t}(12 ; 15)$ pro-B-cell lymphomas in SCID $53^{-/-}$mice involves a lesion in the $\mathrm{V}(\mathrm{D}) \mathrm{J}$ recombinase, suggesting that translocations may arise as a result of aberrant $\mathrm{IgH}$ rearrangement in pro-B cells. By introducing a Rag2-null mutation into the SCID $p 53^{-/-}$strain, we tested whether initiation of $\mathrm{V}(\mathrm{D}) \mathrm{J}$ recombination was a required step in oncogenesis. To exclude background effects, 3 cohorts of mice were derived from this interbreeding, including $p 53^{-/-}\left(S C I D^{w t / w t}, \mathrm{Rag}_{-2^{+/-}}\right.$, or $\left.\mathrm{Rag}^{-2^{+/+}} ; n=13\right)$,

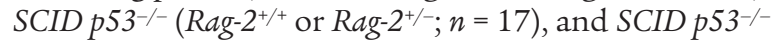
Rag-2-/- $(n=14)$. Mice were observed for tumor development, and tumor phenotype was characterized by flow cytometry as described above. Two separate analyses were performed using survival data for each cohort, considering mortality due to disseminated pro-B-cell lymphoma as well as mortality from all tumor types (Figure 4).

Consistent with previous reports and our earlier experiments, lymphomas developed in $100 \%$ of SCID $p 53^{-/-}$ mice at a median of 8 weeks. All of these tumors were disseminated $\mathrm{B} 220^{+} \mathrm{CD} 19^{+} \mathrm{IgM}^{-}$lymphomas. In the p53-/- cohort, no disseminated pro-B-cell lymphomas were observed during a period of follow-up ranging from 6 to 33 weeks, although 1 animal developed a localized enlargement of a cervical lymph node containing a mature B-cell tumor $\left(\mathrm{B} 220^{+} \mathrm{CD} 19^{+} \operatorname{IgM}^{+}\right)$. In the SCID 


\section{Figure 4}

Rag-2-null mutation suppresses pro-B-cell lymphomas in SCID p53-1mice. (a) Kaplan-Meier survival analysis of mortality due to disseminated pro-B-cell lymphomas in mice derived from a common background. Disseminated pro-B-cell tumors occurred in all of the SCID $p 53^{-/-}$mice $(n=17)$ but were not observed in either the $p 53^{-/-}$cohort $(n=13)$ or in the SCID $p 53^{-/-}$Rag-2-/- cohort $(n=14)$. Follow-up intervals for individual mice are indicated for the $p 53^{-1-}$ (circles) and SCID

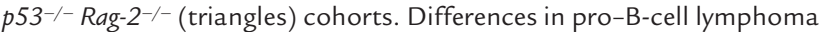
mortality between SCID $p 53^{-/-}$and either $p 53^{-/-}$or SCID $p 53^{-/-}$Rag- $2^{-/-}$ cohorts were highly statistically significant $(P<0.001)$ using the Wilcoxon log rank test. (b) Survival analysis for overall tumor mortality. Differences between the SCID $p 53^{-/-}$and either the $p 53^{-/-}$or SCID $p 53^{-1-}$ Rag-2-/- cohorts were statistically significant using the log rank test $(P$ $<0.001)$. Differences in overall tumor mortality between the $p 53^{-/-}$and $S C I D p 53^{-/-}$Rag- $2^{-/-}$cohorts were not statistically significant $(P>0.40)$.

p53-/- Rag- $^{-2^{--}}$cohort, which was observed for a period ranging from 6 to 22 weeks, no disseminated B-lineage lymphomas were identified. These differences were statistically significant $(P<0.001)$. Analysis of overall tumor mortality demonstrated that the SCID $p 53^{-/-}$cohort had the shortest median survival ( 8 weeks), whereas the median survival of the $p 53^{-/-}$and SCID $p 53^{-/-}$Rag- $2^{-/-}$ cohorts was greater (23 and 18 weeks, respectively). Statistical comparisons indicated that the overall survival of the SCID $\mathrm{p5}^{-/-}$cohort was significantly less than either of the other groups of mice $(P<0.001)$, whereas survival differences between the $p 53^{-/-}$and SCID $p 53^{-/-}$Rag $-^{-2^{-1}}$ cohorts were not statistically significant $(P>0.40)$.

The occurrence of localized thymic lymphomas was

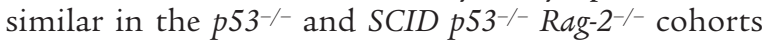
(Table 3). Phenotypically, the majority of SCID $p 53^{-/-}$Rag$2^{-/-}$lymphomas expressed CD90, CD4, and CD8, and lacked TCR- $\alpha \beta, C D 3$, and B-cell markers. One thymic tumor expressed CD90 as well as the B-cell markers B220 and CD19. Sarcomas were also seen in both the $p 53^{-/-}$ and SCID $p 53^{-/-}$Rag-2-/- mice. Thymic lymphomas and sarcomas were not observed in SCID $p 53^{-/-}$mice, likely reflecting the early mortality from disseminated pro-Bcell tumors (Figure 4b). These data indicate that initiation of $\mathrm{V}(\mathrm{D}) \mathrm{J}$ recombination is a required element in the oncogenic pathway leading to disseminated $\mathrm{t}(12 ; 15)$ pro-B-cell lymphomas in SCID $\mathrm{p5}^{53^{-/-}}$mice, but not to the thymic lymphomas and sarcomas characteristic of the $p 53^{-/-}$background.

\section{Discussion}

Chromosome translocations involving antigen receptor genes represent a common pathway for lymphoid oncogenesis and likely represent an early and critical genetic change in the evolution of these malignancies $(1,3,4)$. The hypothesis that these translocations involve $V(D) J$ recombination has been inferred from the sequences of cloned breakpoints. The evidence appears most consistent in the $\mathrm{t}(14 ; 18)$ translocations found in most cases of follicular Bcell lymphomas, which juxtapose the $B c l-2$ proto-oncogene with 1 of the JH segments, and in t(7;9) of T-cell lymphoblastic lymphoma/leukemia, which involves breaks flanking the D and J segments of the TCR- $\beta$ gene on chromosome $7(4,8,9,12,42)$. Other features of $\mathrm{V}(\mathrm{D}) \mathrm{J}$ recom-

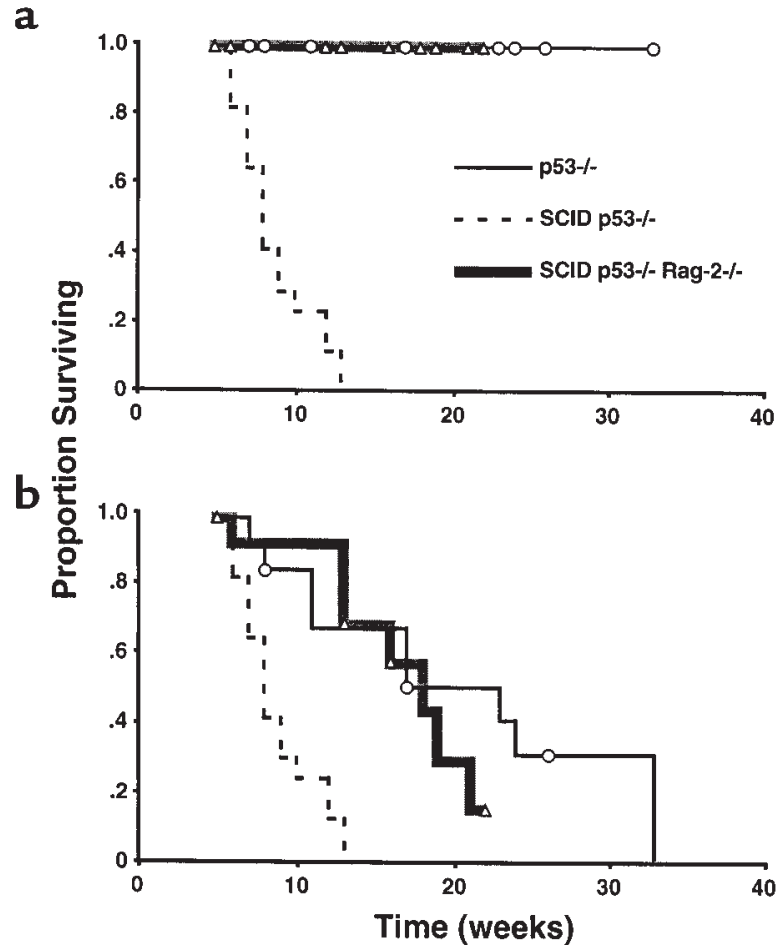

bination, including cryptic RSS on partner chromosomes and $\mathrm{N}$-nucleotide additions, have also been reported at translocation breakpoints (reviewed in ref. 4). Despite the resemblance of some breakpoints to $\mathrm{V}(\mathrm{D}) \mathrm{J}$ joints, much of the available data on breakpoint sequences do not strictly conform to the attributes of $\mathrm{V}(\mathrm{D}) \mathrm{J}$ recombination, indicating that most translocations do not arise from a normal V(D)J reaction. Nevertheless, the consistent participation of antigen receptor loci in lymphoma translocations suggests that components of the $V(D) J$ recombinase may participate in creating the conditions for aberrant chromosome joining.

The recurrent involvement of $\mathrm{t}(12 ; 15)$ in pro-B-cell lymphomas arising in SCID $p 53^{-/-}$mice defines a genetic pathway leading reproducibly to antigen receptor-locus translocations. The fact that a Rag-2-null mutation suppressed the development of $\mathrm{t}(12 ; 15)$ lymphomas in the SCID $p 53^{-/-}$ background suggests that the translocations occur as a result of aberrant rejoining of $\operatorname{IgH}$ loci cleaved during attempted $\mathrm{V}(\mathrm{D}) \mathrm{J}$ recombination at the pro-B-cell stage. An

\section{Table 3}

Comparison of tumor types developing in 3 cohorts of mice derived from a common background

\begin{tabular}{|c|c|c|c|}
\hline Tumor type & $\begin{array}{c}\text { SCID } p 53^{-/-} \\
\quad(n=17)\end{array}$ & $\begin{array}{l}p^{53^{-/-}} \\
(n=13)\end{array}$ & $\begin{array}{c}\text { SCID } p 53^{-/} \\
\text {Rag-2-/- } \\
(n=14)\end{array}$ \\
\hline Disseminated pro-B-cell lymphoma & $17(100)$ & $0(0)$ & $0(0)$ \\
\hline Thymic lymphoma & $0(0)$ & $5(38)$ & $6(43)$ \\
\hline Localized IgM+ B-cell lymphoma & $0(0)$ & $1(7.7)$ & $0(0)$ \\
\hline Sarcoma & $0(0)$ & $3(23)$ & $3(21)$ \\
\hline
\end{tabular}

Lymphoid tumors were classified by flow cytometry, and sarcomas by gross inspection and histology. The number of mice (percent of cohort) developing each tumor type is given. 
alternative interpretation is that oncogenesis is developmentally specific and requires Rag- 2 for maturation of $B$ cells to a susceptible stage. B-cell progression from the pro- $\mathrm{B}$ to pre- $\mathrm{B}$ stage is signaled by productive rearrangement of $\mathrm{C} \mu$ and expression in the context of other components of the pre-B-cell receptor (37). A block in B-cell development at the pro-B stage occurs in both Rag- $2^{--}$and SCID mice, although in the latter strain, there is some leakiness in T-and B-cell development because of the infrequent assembly of $\mathrm{V}(\mathrm{D}) \mathrm{J}$ joints. In all the SCID $p 53^{-/-}$ tumors tested, we found no evidence for surface or cytoplasmic $\mu$-chain expression. Furthermore, in all $4 \mathrm{t}(12 ; 15)$ lymphomas in which IgH rearrangements could be characterized, we definitively ruled out productive $V(D) J$ rearrangement on both IgH alleles. Thus, the cellular target for oncogenic transformation in SCID $p 53^{-/-}$mice could not have passed the pro-B-cell stage.

Following cleavage of antigen receptor DNA in SCID mice, the inability to efficiently rejoin DNA ends could potentially lead to illegitimate recombination. In SCID thymocytes, p53 is activated by DNA breaks, leading to early apoptosis $(33,43)$. In the absence of p53 function, SCID pro-B cells may survive longer, permitting Ragmediated DNA breaks at the IgH locus to be rejoined by activities other than the DNA-PK complex. Such alternative DNA repair pathways may be less stringent in preferentially rejoining antigen receptor DNA ends to each other than in involving another locus, leading to a higher frequency of chromosome translocations. In p53-deficient mice, which have normal lymphoid development, presence of the DNA-PK complex may ensure efficient rejoining of DNA breaks during $\mathrm{V}(\mathrm{D}) \mathrm{J}$ recombination so that unresolved DNA breaks are relatively rare. Our data confirm previous observations that thymic lymphomas commonly occurring in $p 53^{-/-}$mice are not associated with recurrent chromosome translocations (44). The finding that thymic lymphomas occurred in SCID $p 53^{-/-}$Rag- $2^{-1-}$ mice with frequency similar to that of $p 53^{-/-}$mice also agrees with previous work, showing that $p 53^{-/-}$thymic tumors are not suppressed by Rag- 1 or Rag- 2 mutations (44-46). Thus, for some types of lymphoma, p53 contributes to oncogenesis in a manner that is independent of chromosome translocations involving antigen receptor genes. In SCID $\mathrm{p} 53^{-/-}$mice, the $\mathrm{p} 53$ lesion may, therefore, contribute to the high incidence of pro-B-cell lymphoma by failing to prevent chromosome translocations, by facilitating downstream genetic changes, or both.

The hypothesis that cellular responses to DNA damage suppress oncogenic side reactions of the $V(D) J$ recombinase is supported by the association between genetic conditions affecting these pathways and predisposition to lymphoid malignancies. Patients with ataxia-telangiectasia, the Nijmegen breakage syndrome, and Bloom's syndrome all exhibit abnormal cellular responses to DNA damage and have a high incidence of non-Hodgkin's lymphomas (47-50). Lymphomas in ataxia-telangiectasia patients are most commonly T-cell derived and involve translocations with TCR loci on chromosomes 7 or 14 (47). This observation, and the fact that translocation-associated lymphomas in SCID $p 53^{-/-}$mice exclusively involved the B-cell lineage, suggests that the spectrum of critical elements for tumor suppression during antigen receptor rearrangement may differ in the various lymphoid tissues. Advances in understanding the cellular functions of lymphoma tumor suppressors may shed more light on this issue.

Targeted genomic instability is an essential process in diversification of the antigen receptor repertoire and occurs in 3 distinct forms in the B-cell lineage. In addition to VDJ recombination, immune responses induce germinal-center $\mathrm{B}$ cells to undergo isotype class-switch recombination within the IgH locus, as well as somatic hypermutation of immunoglobulin variable regions. Class-switch recombination has been implicated in generating $t(8 ; 14)$ in nonendemic Burkitt's lymphoma, juxtaposing switch regions lying upstream of constant-region exons with c-myc. In mice, plasmacytomas induced by mineral oil are associated with $\mathrm{t}(12 ; 15)$, placing the $\mathrm{c}-m y c$ locus on chromosome 15 into the switch segments of $3^{\prime} \operatorname{IgH}$ constant-region genes $(13,51,52)$. This mechanism is unlikely to be involved in mediating the $t(12 ; 15)$ in $S C I D p 53^{-/-}$lymphomas, given that Rag- 2 is required for generation of these tumors but is not needed for class switching $(53,54)$. Recent data indicate that somatic hypermutation also involves DNA breaks and may contribute to some IgH translocations that occur within the variable region $(55,56)$. It has also been determined that Rag-1 and Rag- 2 are reactivated in germinal-center B cells, where immunoglobulin genes may undergo additional $\mathrm{V}(\mathrm{D}) \mathrm{J}$ recombination events (57-60). The apparent genetic plasticity of immunoglobulin genes in the germinal center correlates with a postulated germinal-center origin for the majority of B-cell lymphomas and suggests that, in many cases, chromosome translocations may arise in the course of immune responses $(61,62)$.

Genetic instability at antigen receptor loci represents the distinguishing feature of vertebrate immunity and conflicts with the cellular paradigm of preserving genomic integrity. An important question regarding the mechanism of $\mathrm{V}(\mathrm{D}) \mathrm{J}$ recombination and other forms of antigen receptor revision is how these processes are normally confined to intended targets. Our data suggest that this confinement involves 2 essential components. First, efficient rejoining of DNA breaks may minimize cellular exposure to free DNA ends. This is likely facilitated by the coupling of Rag-mediated cleavage events and the formation of a synaptic complex that activates rejoining (63). Second, activation of DNA damage-sensing pathways by unresolved DNA breaks may either kill cells or otherwise prevent the activity of nonspecific repair pathways. Genetic factors or environmental exposures that interfere with either of these processes may increase the frequency of chromosome translocations and contribute to the development of lymphomas.

\section{Acknowledgments}

We thank Roy Riblet for generously providing us with the IgH BAC, Barbara Malynn for the IgH locus probes, and Frederick Alt for supplying Rag-2-/- mice. We are grateful to Mark Groudine and Patrick Concannon for reviewing the manuscript. This work was supported in part by grants from the National Institutes of Health (to G.J. Vanasse, D.M. Willerford, and C.M. 
Disteche) and the University of Washington Royalty Research Fund (to D.M. Willerford).

1. Rabbitts, T.H. 1994. Chromosomal translocations in human cancer. Nature. 372:143-149.

2. Showe, L.C., and Croce, C.M. 1987. The role of chromosomal translocations in B- and T-cell neoplasia. Annu. Rev. Immunol. 5:253-277.

3. Korsmeyer, S.J. 1992. Chromosomal translocations in lymphoid malignancies reveal novel proto-oncogenes. Annu. Rev. Immunol. 10:785-807.

4. Tycko, B., and Sklar, J. 1990. Chromosomal translocations in lymphoid neoplasia: a reappraisal of the recombinase model. Cancer Cells. 2:1-8.

5. Alt, F.W., et al. 1992. VDJ recombination. Immunol. Today. 13:306-314.

6. Roth, D.B., Lindahl, T., and Gellert, M. 1995. Repair and recombination. How to make ends meet. Curr. Biol. 5:496-499.

7. Harriman, W., Volk, H., Defranoux, N., and Wabl, M. 1993. Immunoglobulin class switch recombination. Annu. Rev. Immunol. 11:361-384.

8. Bakhshi, A., et al. 1985. Cloning the chromosomal breakpoint of $t(14 ; 18)$ human lymphomas: clustering around $\mathrm{J}_{\mathrm{H}}$ on chromosome 14 and near a transcriptional unit on 18. Cell. 41:899-906.

9. Tsujimoto, Y., Gorham, J., Cossman, J., Jaffe, E., and Croce, C.M. 1985. The $\mathrm{t}(14 ; 18)$ chromosome translocations involved in B-cell neoplasms result from mistakes in VDJ joining. Science. 22:1390-1393.

10. Taub, R., et al. 1982. Translocation of the c-myc gene into the immunoglobulin heavy chain locus in human Burkitt lymphoma and murine plasmacytoma cells. Proc. Natl. Acad. Sci. USA. 79:7837-7841.

11. Haluska, F.G., Finver, S., Tsujimoto, Y., and Croce, C.M. 1986. The t $(8 ; 14)$ chromosomal translocation occurring in B-cell malignancies results from mistakes in V-D-J joining. Nature. 325:158-161.

12. Cleary, M.L., and Sklar, J. 1985 . Nucleotide sequence of a t $(14 ; 18)$ chromosomal breakpoint in follicular lymphoma and demonstration of a breakpoint cluster region near a transcriptionally active locus on chromosome 18. Proc. Natl. Acad. Sci. USA. 82:7439-7443.

13. Cory, S., Gerondakis, S., and Adams, J.M. 1983. Interchromosomal recombination of the cellular oncogene c-myc with the immunoglobulin heavy chain locus in murine plasmacytomas is a reciprocal exchange. $E M B O J$. 2:697-703.

14. Schatz, D.G., Oettinger, M.A., and Schlissel, M.S. 1992. V(D)J recombination: molecular biology and regulation. Annu. Rev. Immunol. 10:359-383.

15. Oettinger, M.A., Schatz, D.G., Gorka, C., and Baltimore, D.G. 1990. RAG1 and RAG-2, adjacent genes that synergistically activate $V(D) J$ recombination. Science. 248:1517-1522.

16. van Gent, D.C., et al. 1995. Initiation of V(D)J recombination in a cell-free system. Cell. 81:925-934.

17. McBlane,J.F., et al. 1995. Cleavage at a V(D)J recombination signal requires only RAG1 and RAG2 proteins and occurs in two steps. Cell. 83:387-395.

18. Mombaerts, P., et al. 1992. RAG-1 deficient mice have no mature B and T lymphocytes. Cell. 68:869-877.

19. Shinkai, Y., et al. 1992. RAG-2 deficient mice lack mature lymphocytes owing to inability to initiate V(D)J rearrangement. Cell. 68:855-867.

20. Schwartz, K., et al. 1996. RAG mutations in human B cell-negative SCID. Science. 274:97-99.

21. Taccioli, G.E., and Alt, F.W. 1992. Potential targets for autosomal SCID mutations. Curr. Opin. Immunol. 7:436-440.

22. Roth, D.B., Menetski, J.P., Nakajima, P.B., Bosma, M.J., and Gellert, M. 1992. V(D)J recombination: broken DNA molecules with covalently sealed (hairpin) coding ends in scid mouse thymocytes. Cell. 70:983-991.

23. Bosma, M.J., and Carroll, A.M. 1991. The SCID mouse mutant: definition, characterization, and potential uses. Annu. Rev. Immunol. 9:323-350.

24. Blunt, T., et al. 1995. Defective DNA-dependent protein kinase activity is linked to $V(D) J$ recombination and DNA repair defects associated with the murine scid mutation. Cell. 80:813-823.

25. Kirchgessner, C.U., et al. 1995. DNA-dependent protein kinase (p350) as a candidate gene for the murine SCID defect. Science. 267:1178-1183.

26. Peterson, S.R., et al. 1995. Loss of the catalytic subunit of the DNA-dependent protein kinase in DNA double-strand-break-repair mutant mammalian cells. Proc. Natl. Acad. Sci. USA. 92:3171-3174.

27. Kastan, M.B., et al. 1992. A mammalian cell cycle checkpoint pathway utilizing p53 and GADD45 is defective in ataxia-telangiectasia. Cell. 71:587-597.

28. Clarke, A.R., et al. 1993. Thymocyte apoptosis induced by p53-dependent and independent pathways. Nature. 362:849-852

29. Lowe, S., Schmitt, E.M., Smith, S.W., Osborne, B.A., and Jacks, T. 1993. Nature. 362:847-851.

30. Donehower, L.A., et al. 1992. Nature. 356:215-221.

31. Nacht, M., et al. 1996. Mutations in the p53 and SCID genes cooperate in tumorigenesis. Genes Dev. 10:2055-2066.

32. Bogue, M.A., Zhu, C., Aguilar-Cordova, E., Donehower, L.A., and Roth, D.B. 1996. p53 is required for both radiation-induced differentiation and rescue of V(D)J rearrangement in scid mouse thymocytes. Genes Dev. 10:553-565.

33. Guidos, C.J., et al. 1996. V(D)J recombination activates a p53-dependent
DNA damage checkpoint in scid lymphocyte precursors. Genes Dev. 10:2038-2054

34. Malynn, B.A., et al. 1988. The scid defect affects the final step of the immunoglobulin VDJ recombinase mechanism. Cell. 54:453-460.

35. Shi, Y.P., et al. 1997. FISH probes for mouse chromosome identification. Genomics. 45:42-47.

36. Edelhoff, S.E., et al. 1994. Mapping of two genes encoding members of a distinct subfamily of MAX interacting proteins: MAD to human chromosome 2 and mouse chromosome 6, and MXI1 to human chromosome 10 and mouse chromosome 19. Oncogene. 9:665-668.

37. Willerford, D.M., Swat, W., and Alt, F.W. 1996. Developmental regulation of V(D)J recombination and lymphocyte differentiation. Curr. Opin. Genet. Dev. 6:603-609.

38. Schuler, W., et al. 1986. Rearrangement of antigen receptor genes is defective in mice with severe combined immune deficiency. Cell. 46:963-972.

39. Donehower, L.A., et al. 1995. Effects of genetic background on tumorigenesis in p53-deficient mice. Mol. Carcinog. 14:16-22.

40. Yunis, J.J., et al. 1982. Distinctive chromosomal abnormalities in histologic subtypes of non-Hodgkin's lymphoma. N. Engl. J. Med. 307:1231-1236.

41. Cook, G.P., et al. 1994. A map of the human immunoglobulin VH locus completed by analysis of the telomeric region of chromosome 14q. Nat. Genet. 7:162-168.

42. Tycko, B., Reynolds, T.C., Smith, S.D., and Sklar, J. 1989. Consistent breakage between consensus recombinase heptamers of chromosome 9 DNA in a recurrent chromosomal translocation of human T cell leukemia. J. Exp. Med. 169:369-377.

43. Diamond, R.A., Ward, S.B., Owada-Makabe, K., Wang, H., and Rothenberg, E.V. 1997. Different developmental arrest points in Rag-2-/- and SCID thymocytes on two genetic backgrounds. J. Immunol. 158:4052-4064.

44. Liao, M.-J., et al. 1998. No requirement for V(D)J recombination in p53-deficient thymic lymphoma. Mol. Cell. Biol. 18:3495-3501.

45. Mombaerts, P., Terhorst, C., Jacks, T., Tonegawa, S., and Sancho, J. 1995. Characterization of immature thymocyte lines derived from $\mathrm{T}$-cell receptor or recombination activating gene 1 and 553 double mutant mice. Proc. Natl. Acad. Sci. USA. 92:7420-7424.

46. Nacht, M., and Jacks, T. 1998. V(D)J recombination is not required for the development of lymphoma in p53-deficient mice. Cell Growth Differ. 9:131-138.

47. Lavin, M.F., and Shiloh, Y. 1997. The genetic defect in ataxia-telangiectasia. Annu. Rev. Immunol. 15:177-202.

48. Varon, R., et al. 1998. Nibrin, a novel DNA double-strand break repair protein is mutated in Nijmegen breakage syndrome. Cell. 93:467-476.

49. Ellis, N.A., et al. 1995. The Bloom's syndrome gene product is homologous to RecQ helicases. Cell. 83:655-666.

50. German, J. 1997. Bloom's syndrome. XX. The first 100 cancers. Cancer Genet. Cytogenet. 93:100-106.

51. Gerondakis, S., Cory, S., and Adams, J.M. 1984. Translocation of the myc cellular oncogene to the immunoglobulin heavy chain locus in murine plasmacytomas is an imprecise reciprocal exchange. Cell. 36:973-982.

52. Potter, M., and Wiener, F. 1992. Plasmacytomagenesis in mice: model of neoplastic development dependent upon chromosomal translocations. Carcinogenesis. 13:1681-1697.

53. Rolink, A., Melchers, F., and Andersson, J. 1996. The SCID but not the RAG-2 gene product is required for $\mathrm{S}$ mu-S epsilon heavy chain class switching. Immunity. 5:319-330.

54. Lansford, R., Manis, J.P., Sonoda, E., Rajewsky, K., and Alt, F.W. 1998. Ig heavy chain class switching in Rag-deficient mice. Int. Immunol. 10:325-332.

55. Sale, J.E., and Neuberger, M.S. 1998. TdT-accessible breaks are scattered over the immunoglobulin $\mathrm{V}$ domain inn a constitutively hypermutating $\mathrm{B}$ cell line. Immunity. 9:859-869.

56. Goossens, T., Klein, U., and Kuppers, R. 1998. Frequent occurrence of deletions and duplications during somatic hypermutation: implications for oncogene translocations and heavy chain disease. Proc. Natl. Acad. Sci. USA. 95:2463-2468.

57. Han, S., Zheng, B., Schatz, D.G., Spanopoulou, E., and Kelsoe, G. 1996. Neoteny in lymphocytes: Rag1 and Rag2 expression in germinal center B cells. Science. 274:2094-2097.

58. Han, S., et al. 1997. V(D)J recombinase activity in a subset of germinal center B lymphocytes. Science. 278:301-305.

59. Hikida, M., et al. 1996. Reexpression of Rag-1 and Rag-2 genes in activated mature mouse B cells. Science. 274:2092-2094.

60. Papavasiliou, F., et al. 1997. V(D)J recombination in mature B cells: a mechanism for altering antibody responses. Science. 278:298-301.

61. Harris, N.L., et al. 1994. A revised European-American classification of lymphoid neoplasms: a proposal from the International Lymphoma Study Group. Blood. 84:1361-1392.

62. Klein, U., et al. 1998. Somatic hypermutation in normal and transformed B cells. Immunol. Rev. 162:261-280.

63. Hiom, K., and Gellert, M. 1998. Assembly of a $12 / 23$ paired signal complex: a critical control point in V(D)J recombination. Mol. Cell. 1:1011-1019 